\title{
Data: The Lifeblood of the Public Health Response to COVID-19
}

M ore than 18 months after SARS-CoV-2 was first detected in the United States, we are only now beginning to have the data to properly assess the effect of the COVID-19 pandemic in this country. The absence of good, clear data has fed misinformation and been fertile ground for disinformation (1). In the early days of the pandemic, the data gap led many to underestimate the threat posed by the virus and oppose public health measures pending further study (2). Fortunately, public health officials recognized the threat signaled in preliminary data from China that suggested that the virus was both highly transmissible and virulent. Acting on the limited evidence we did have, the nation pushed forward with control measures, albeit inconsistently.

Eighteen months later, we have begun to fill in some of the gaps in our knowledge of the clinical effect of this virus. We have recorded more than 660000 U.S. deaths due to COVID-19 (3), a tally that is both shocking and almost certainly an underestimate, given analysis of excess mortality and the inadequacy of testing that remains a constant feature of this pandemic. A growing body of evidence has highlighted the unconscionably disparate effect of the pandemic on Black and Hispanic Americans (4). These data are crucial to our ongoing work to curb the pandemic, but they also leave important questions unanswered, particularly around clinical burden.

In their article, Reif and colleagues (5) make a critically important contribution to our understanding of the burden of mortality due to the COVID-19 pandemic. Their rigorous analysis focused on years of life lost (YLLs), a standard measure of mortality that allows a better assessment of the true cost of the pandemic by taking account of the life expectancy of those who died during its first year in the United States (6).

Using 3 modeling approaches to assess excess mortality due to COVID-19, these researchers estimated that the pandemic accounted for more than 9 million YLLs compared with if it had never happened (6). This is a sobering finding in its own right and an important contribution to the ongoing work to quantify the pandemic-related suffering Americans have endured. Years of life lost, of course, is only 1 measure of suffering. We must also consider others, such as the short-term disability caused by illness and the burden of persisting symptoms after acute SARS-CoV-2 infection and other sequelae. However, this analysis focusing on excess mortality across different populations yields crucial findings that deepen our understanding of the pandemic and its effect in the United States.

First, although most U.S. deaths from COVID-19 occurred in those older than 65 years (7), Reif and colleagues' analysis shows that more than half of qualityadjusted life-years (QALYs) were lost by those aged between 25 and 64 years (5). Throughout the pandemic, some have asserted that the primary effect of COVID-19 is limited to older Americans, a view that is both cavalier in its treatment of those Americans and factually inaccurate.
Second, this analysis helps us better understand the role of key risk factors in COVID-19 mortality, indicating that although dementia is the most influential risk factor, it affects only a small number of Americans; most Americans have other far more common risk factors for COVID-19 death, including diabetes, obesity, and smoking history. Furthermore, Reif and colleagues found that the effect of COVID-19 is by no means limited to people with comorbidities. Indeed, one third of those who died of COVID-19 would have lived as long as or longer than their peers of the same age, sex, and race or ethnicity. In other words, although the pandemic certainly exploited the vulnerabilities of frail Americans with comorbidities, Americans fell prey to the worst disease outbreak in a century without regard to their prior health status. The researchers concluded that COVID-19 tends to target individuals with close-to-average life expectancy for their population subgroup.

This analysis also lays to rest the myth that SARS-CoV-2 similarly threatens all Americans regardless of race or socioeconomic status. Reif and colleagues found that older Black and Hispanic Americans lost twice as many QALYs per person as their White peers, whereas those between 25 and 64 years lost 3 times as many as White Americans of the same age (5). This disparate racial effect is likely due to a range of factors, including that Black and Hispanic Americans are more likely than their White peers to be employed in jobs that cannot be done remotely, to take public transit, to live in multigenerational homes, and to have poor access to health care. As the current analysis shows, the pandemic underscores the United States' failure to address racial inequities in social determinants of health, access to care, and policy priorities (7).

As important as this study is, it is an incomplete analysis of the burden of American suffering as the pandemic continues. The study analyzed deaths due to COVID-19 through the middle of March 2021. In the months since, more than 120000 Americans have died of COVID-19, and it is likely that the burden of disease has shifted to a younger population as older Americans have generally embraced vaccination. In addition, YLLs due to the pandemic may be decreasing more slowly than raw mortality data suggest. Furthermore, measuring mortality yields an incomplete picture of the pandemic's effect. Those who survive COVID-19 often face a long road to recovery and may endure chronic symptoms and disability $(8,9)$. Even if such conditions do not lead to YLLs, they must be included in any thorough analysis that seeks to examine the pandemic's effects on health and well-being.

Data are the lifeblood of any public health response. Unfortunately, during the first year of the pandemic, our public health system simply failed to report basic, coherent data on infections, hospitalizations, and deaths-a void partially filled by startup organizations like the COVID Tracking Project (10). This year, the data have improved, but important gaps remain, particularly around breakthrough infections and the burden of hospitalization. To 


\section{EDITORIAL}

guide our response to this pandemic and prepare for future ones, we must continue the hard work of collecting and analyzing data to understand the true scope of COVID-19's effect.

Ashish K. Jha, MD, MPH

Brown University School of Public Health

Providence, Rhode Island

Disclosures: The author has reported no disclosures of interest. The form can be viewed at www.acponline.org/authors/icmje /ConflictOflnterestForms.do?msNum=M21-3725.

Corresponding Author: Ashish K. Jha, MD, MPH, Dean, Brown University School of Public Health, 121 South Main Street, Box GS121-3, Brown University, Providence, RI 02912; e-mail, ashish_jha@ brown.edu.

Ann Intern Med. doi:10.7326/M21-3725

\section{References}

1. Butcher P. COVID-19 as a turning point in the fight against disinformation. Nat Electron. 2021;4:7-9. doi:10.1038/s41928-02000532-2

2. Schneider EC. Failing the test - the tragic data gap undermining the U.S. pandemic response. N Engl J Med. 2020;383:299-302. [PMID: 32412704] doi:10.1056/NEJMp2014836
Data: The Lifeblood of the Public Health Response to COVID-19

3. Centers for Disease Control and Prevention. COVID Data Tracker. 2021. Accessed athttps://covid.cdc.gov/covid-data-tracker/\#trends_ dailydeaths on 14 September 2021.

4. Lopez L 3rd, Hart LH 3rd, Katz MH.. Racial and ethnic health disparities related to COVID-19. JAMA. 2021;325:719-720. [PMID: 33480972] doi:10.1001/jama.2020.26443

5. Reif J, Heun-Johnson $H$, Tysinger B, et al. Measuring the COVID19 mortality burden in the United States. A microsimulation study. Ann Intern Med. 2020. [Epub ahead of print]. doi:10.7326/M21-2239 6. Devleesschauwer B, McDonald SA, Speybroeck N, et al. Valuing the years of life lost due to COVID-19: the differences and pitfalls [Letter]. Int J Public Health. 2020;65:719-720. [PMID: 32691080] doi:10.1007 /s00038-020-01430-2

7. Freed M, Cubanski J, Neuman T, et al. What share of people who have died of COVID-19 are 65 and older - and how does it vary by state? Kaiser Family Foundation. 24 July 2020. Accessed at www.kff. org/coronavirus-covid-19/issue-brief/what-share-of-people-who-havedied-of-covid-19-are-65-and-older-and-how-does-it-vary-by-state on 14 September 2021.

8. Lerner AM, Robinson DA, Yang L, et al. Toward understanding COVID-19 recovery: National Institutes of Health workshop on postacute COVID-19. Ann Intern Med. 2021;174:999-1003. [PMID: 33780290] doi:10.7326/M21-1043

9. Nehme M, Braillard O, Chappuis F, et al. Prevalence of symptoms more than seven months after diagnosis of symptomatic COVID-19 in an outpatient setting. Ann Intern Med. 2021. [PMID: 34224254] doi:10.7326/M21-0878

10. The COVID Tracking Project. The Atlantic Monthly Group. 2021. Accessed athttps://covidtracking.com/data on 14 September 2021. 